\title{
Boundedness and Asymptotic Behaviour of Solutions of a Second Order Nonlinear Differential Equation
}

\author{
Maher Nazmi Qarawani \\ Department of Mathematics, AlQuds Open University, Palestine \\ E-mail: mkerawani@qou.edu
}

Received: April 6, 2012 Accepted: May 8, 2012 Online Published: May 29, 2012

doi:10.5539/jmr.v4n3p121 URL: http://dx.doi.org/10.5539/jmr.v4n3p121

\begin{abstract}
This paper considers asymptotic behaviour of solutions of a nonlinear differential equation of second order where the coefficient of nonlinearity is a bounded function for arbitrarily large values of $x$ in $\mathbb{R}$. Here we obtained sufficient conditions for boundedness, convergence of solutions to zero as $x \rightarrow \infty$, and unboundedness of solutions.
\end{abstract}

Keywords: Boundedness, Asymptotic behaviour, Nonlinear, Second-order, Differential equation

\section{Introduction}

Mathematical modelling of most natural, scientific and industrial phenomena is based on nonlinear differential equations which are not easily solvable. Therefore, the determination of behaviour of solutions has attracted the attention of researchers over years (Ademole \& Arawomo, 2011).

The monographs by many authors contain good results, including existence Theorems, asymptotic behaviour of solutions stability theory and oscillation theory.

Waltman (1964) proved that if $a(t)$ is continuous and $\int_{0}^{\infty} t^{2 n+1}|a(t)| d t$ then the equation $x^{\prime \prime}+a(t) x^{2 n+1}=0$, where $\mathrm{n}$ is a nonnegative integer, has a solution $x(t)$ satisfying $\lim _{t \rightarrow \infty} \frac{x(t)}{t}=a \neq 0$. Moreover, the solutions of that equation have asymptotic form

$$
x(t)=a t+b+O(1), \mathrm{a}, \mathrm{b} \text { constants }
$$

Kiguradze (1965) had obtained sufficient conditions for existence solutions that tend to $\pm \infty$ as $t \rightarrow \infty$ of a nonlinear differential equation of Emden-Fowler type

$$
u^{\prime \prime}-a(t)|u|^{n} \operatorname{sgnu}=0, \text { where } a(t)>0 \text { and } n>1 .
$$

The study (Tong, 1982) showed that the nonlinear differential equation $u^{\prime \prime}+f(t, u) \operatorname{sgn} u=0$ has solutions which are asymptotic to $a+b t$ where $\mathrm{a}, \mathrm{b}$ are constants and $b \neq 0$.

Sufficient criteria of integral type were obtained by Mingareilli and Sadarangani in (2007) for non-oscillation and asymptotic behaviour of solutions of nonlinear differential equation

$$
u^{\prime \prime}+F(x, y(x))=g(x)
$$

They proved the existence of a positive asymptotically linear solution $y(x)=a x+b+O(1)$, as $x \rightarrow \infty$. While Lipovan in her study (2003) has obtained for a class of second order nonlinear differential equations, sufficient conditions that are presented to ensure that some, respectively all solutions are asymptotic to lines. More explicitly, she has given sufficient conditions of integral type guaranteeing that for all $a, b \in R$, there is a solution to the equation $u^{\prime \prime}=f(t, u), t \geq 1$ such that $u(t)=a t+b+o(1)$ as $t \rightarrow \infty$.

Briefly the asymptotic behaviour of solutions of nonlinear differential equation of second order has been widely studied by Wong (1975), Trench (1976), Kusano, Naito and Usam (1986), Constantin (2000), Hovhannisyan (2004), Saker (2006) and many others.

Thus our study is a continuation of preceding contributions to qualitative theory of differential equations. The aim of this paper is to get sufficient conditions for boundedness, asymptotic convergence to zero as $x \rightarrow \infty$ and unboundedness solutions of the equation (1). In this paper we will impose integral conditions on the nonlinear 
coefficient function $h(x)$. Here we do not impose on $h(x)$ any additional conditions like monotonicity or definiteness of sign.

Motivation for this study comes from the work of Bucur (2006) where a boundedness criterion was obtained for the linear equation

$$
y^{\prime \prime}+y=\alpha(x) y, \text { where } \alpha(x) \rightarrow 0, \text { as } x \rightarrow \infty
$$

It should be note that we apply a similar approach to the one used by Bucur, to establish the boundedness of solutions of nonlinear equation.

In this paper, we consider a nonlinear differential equation of second order

$$
z^{\prime \prime}+p(x) z^{\prime}+q(x) z=h(x)|z|^{\alpha} e^{\left(\frac{\alpha-1}{2}\right) \int p(x) d x} \operatorname{sgn} z, \alpha \in(-1,1) \backslash\{0\},
$$

where $q \in C^{0}\left[x_{0}, x\right], x_{0} \geq 0, h, p \in C^{1}\left[x_{0}, x\right], p(x)>0$ and $h(x)$ is a bounded for all sufficiently large $x$ in $R$.

It should be note here that we may assume that $z>0$, because if $z<0$ we set $z=-u, u>0$. So we will consider in future the equation

$$
z^{\prime \prime}+p(x) z^{\prime}+q(x) z=h(x) z^{\alpha} e^{\left(\frac{\alpha-1}{2}\right) \int p(x) d x}, \alpha \in(-1,1) \backslash\{0\},
$$

\section{Boundedness and Asymptotic Behaviour of Solutions}

Lemma 1 A substitution $z(x)=y(x) \exp \left(-\frac{1}{2} \int p(x) d x\right)$ reduces the equation (2) to the equation (3)

$$
y^{\prime \prime}+y=h(x) y^{\alpha}, \alpha \in(-1,1) \backslash\{0\}
$$

where

$$
q(x)-\frac{1}{4} p^{2}(x)-\frac{1}{2} p^{\prime}(x)=1 .
$$

Proof. Let $z(x)=y(x) \exp \left(-\frac{1}{2} \int p(x) d x\right)$ be given. Then we get

$$
\begin{aligned}
& z^{\prime}(x)=y^{\prime}(x) \exp \left(-\frac{1}{2} \int p(x) d x\right)-\frac{1}{2} p(x) \exp \left(-\frac{1}{2} \int p(x) d x\right) y(x) \\
& z^{\prime \prime}(x)=y^{\prime \prime}(x) \exp \left(-\frac{1}{2} \int p(x) d x\right)-y^{\prime}(x) p(x) \exp \left(-\frac{1}{2} \int p(x) d x\right)+ \\
& +\frac{1}{4} p^{2}(x) \exp \left(-\frac{1}{2} \int p(x) d x\right) y(x)-\frac{1}{2} p^{\prime}(x) \exp \left(-\frac{1}{2} \int p(x) d x\right) y(x)
\end{aligned}
$$

Substituting the expressions $z(x), z^{\prime}(x), z^{\prime \prime}(x)$ in the equation (2) and dividing by $\exp \left(-\frac{1}{2} \int p(x) d x\right)$, then we get the equation (3).

Example 1 Consider the equation

$$
z^{\prime \prime}+\frac{2}{x} z^{\prime}+z=\frac{5 e^{-x} z^{\frac{1}{2}}}{\sqrt{x}}
$$

Here we have $p(x)=\frac{2}{x}, q(x)=1, \alpha=\frac{1}{2}$

$$
\begin{gathered}
z(x)=e^{-\frac{1}{2} \int \frac{2}{x} d x}, y(x)=\frac{y(x)}{x} \\
z^{\prime}(x)=x^{-1} y^{\prime}(x)-x^{-2} y(x), \quad z^{\prime \prime}(x)=x^{-1} y^{\prime \prime}(x)-2 x^{-2} y^{\prime}(x)+2 x^{-3} y(x)
\end{gathered}
$$

Substituting $z(x), z^{\prime}(x), z^{\prime \prime}(x)$ in the equation

$$
\begin{gathered}
x^{-1} y^{\prime \prime}(x)-2 x^{-2} y^{\prime}(x)+2 x^{-3} y(x)+2 x^{-2} y^{\prime}(x)-2 x^{-3} y(x)+\frac{y(x)}{x}=\frac{5 e^{-x} y^{\frac{1}{2}}}{x} \\
y^{\prime \prime}(x)+y(x)=5 e^{-x} y^{\frac{1}{2}}
\end{gathered}
$$

Note that the condition $1+\frac{1}{4} p^{2}(x)+\frac{1}{2} p^{\prime}(x)=1+\frac{1}{4}\left(\frac{2}{x}\right)^{2}+\frac{1}{2}\left(\frac{-2}{x^{2}}\right)=1=q(x)$ holds. 
Theorem 1 Suppose that $h(x)$ is a continuously differentiable function, bounded for all sufficiently large $x \in R$, and that the integral $\int_{x_{0}}^{\infty}\left|h^{\prime}(x)\right| d x$ is convergent then any solution of equation is bounded as $x \rightarrow \infty$.

Proof. Multiplying both sides of the equation (2) by $y^{\prime}$ and integrate the result with respect to $\mathrm{x}$ from some positive $x_{0}$ to $x$

$$
\begin{gathered}
\int_{x_{0}}^{x} y^{\prime}(t) \cdot y^{\prime \prime}(t) d t+\int_{x_{0}}^{x} y(t) \cdot y^{\prime}(t) d t=2 \int_{x_{0}}^{x} h(t) \cdot y^{\alpha}(t) y^{\prime}(t) d t \\
y^{\prime 2}(t)\left|\begin{array}{l}
x \\
x_{0}
\end{array}+y^{2}(t)\right| \begin{array}{l}
x \\
x_{0}
\end{array}=2 \int_{x_{0}}^{x} h(t) \cdot y^{\alpha}(t) y^{\prime}(t) d t \\
y^{\prime 2}(x)-y^{\prime 2}\left(x_{0}\right)+y^{2}(x)-y^{2}\left(x_{0}\right)=2 \int_{x_{0}}^{x} h(t) \cdot y^{\alpha}(t) y^{\prime}(t) d t
\end{gathered}
$$

Integrating by parts the integral on the right-hand side we get

$$
\begin{gathered}
y^{\prime 2}(x)+y^{\prime 2}(x)=y^{\prime 2}\left(x_{0}\right)+y^{2}\left(x_{0}\right)-\frac{2 h\left(x_{0}\right) y^{\alpha+1}\left(x_{0}\right)}{\alpha+1} \\
+\frac{2 h(x) y^{\alpha+1}(x)}{\alpha+1}-\frac{2}{\alpha+1} \int_{x_{0}}^{x} h^{\prime}(t) \cdot y^{\alpha+1}(t) d t
\end{gathered}
$$

Hence

$$
y^{2}(x) \leq y^{\prime 2}(x)+y^{2}(x) \leq A_{x_{0}}+\frac{2|h(x)||y(x)|^{\alpha+1}}{\alpha+1}+\frac{2}{\alpha+1} \int_{x_{0}}^{x}\left|h^{\prime}(t)\right| \cdot|y(t)|^{\alpha+1} d t
$$

where $A_{x_{0}} \geq 0$ is an expression dependent only on $x_{0}$.

Let $M=\max _{x_{0} \leq t \leq x}|y(t)|$, and without loss of generality we may assume that $M \geq a_{0}>0$, otherwise the theorem is proved. Since $h(x)$ is bounded we get

$$
\begin{gathered}
M^{2} \leq A_{x_{0}}+\frac{2 B_{0} M^{\alpha+1}}{\alpha+1}+\frac{2 M^{\alpha+1}}{\alpha+1} \int_{x_{0}}^{x}\left|h^{\prime}(t)\right| d t, \alpha \in(-1,1) \backslash\{0\} \\
M^{1-\alpha} \leq \frac{A_{x_{0}}}{M^{\alpha+1}}+\frac{2 B_{0}}{\alpha+1}+\frac{2}{\alpha+1} \int_{x_{0}}^{x}\left|h^{\prime}(t)\right| d t \leq \frac{A_{x_{0}}}{a_{0}^{\alpha+1}}+\frac{2 B_{0}}{\alpha+1}+\frac{2}{\alpha+1} \int_{x_{0}}^{\infty}\left|h^{\prime}(x)\right| d x
\end{gathered}
$$

Since the integral $\int_{x_{0}}^{\infty}\left|h^{\prime}(x)\right| d x$ converges we obtain

$$
|y(x)| \leq M \leq\left(C_{0}+2 B_{1}+2 D_{0}\right)^{\frac{1}{1-\alpha}} \quad, \alpha \in(-1,1) \backslash\{0\}
$$

Therefore $y(x)$ is bounded for $x \rightarrow \infty$.

Now we give an example illustrating the theorem.

Example 2 Consider the differential equation

$$
y^{\prime \prime}+y=\frac{\sqrt{y}}{x}
$$

We will show that all its solutions are bounded for $x \rightarrow \infty$.

Here $\alpha=\frac{1}{2}, h(x)=\frac{1}{x},|h(x)|=\left|\frac{1}{x}\right| \leq 1$, for $|x| \geq 1$ and $\int_{1}^{\infty}\left|h^{\prime}(x)\right| d x=\int_{1}^{\infty}\left|\frac{-1}{x^{2}}\right| d x=\int_{1}^{\infty} \frac{1}{x^{2}} d x$ converges to 1 .

Applying the same approach used in theorem to this equation, we get,

$$
M^{\frac{1}{2}} \leq \frac{A_{0}}{M^{\frac{3}{2}}}+\frac{2 B_{0}}{\alpha+1}+\frac{2}{\alpha+1} \int_{1}^{x}\left|h^{\prime}(t)\right| d t \leq \frac{A_{0}}{a_{0}}+2+2 \int_{1}^{\infty}\left|h^{\prime}(x)\right| d x
$$

Since $\int_{1}^{\infty}\left|h^{\prime}(x)\right| d x$ converges to 1 , we get

$$
|y(x)| \leq M \leq\left(C_{1}+4\right)^{2} \text {, as } x \rightarrow \infty .
$$

Which completes the proof. 
Theorem 2 Assume that $h(x)$ satisfies the conditions of theorem 1 and $\int_{x_{0}}^{\infty}|h(x)| d x<\infty$, then for any solution $y(x)$ of equation (3) holds the asymptotic formula

$$
y(x)=A \sin \left(x+\omega_{0}\right)+O\left(\int_{x_{0}}^{\infty}|h(x)| d x\right),
$$

where $A, \omega_{0}$ are some constants.

Proof. We treat the right-hand side of the equation (3) as a known function. Then using the method of variations of parameters we get the solution of the equation (3)

$$
\begin{aligned}
y(x) & =c_{1} \sin x+c_{2} \cos x+\int_{x_{0}}^{x} \sin (x-t) y^{\alpha}(t) h(t) d t \\
& =A \sin \left(x+\omega_{0}\right)+\int_{x_{0}}^{x} \sin (x-t) y^{\alpha}(t) h(t) d t
\end{aligned}
$$

Implies that

$$
\left|y(x)-A \sin \left(x+\omega_{0}\right)\right| \leq \int_{x_{0}}^{x}\left|y^{\alpha}(t) h(t)\right| d t
$$

By Theorem 1 the function $y(x)$ is bounded, hence we get

$$
\left|y(x)-A \sin \left(x+\omega_{0}\right)\right| \leq C_{2} \int_{x_{0}}^{x}|h(t)| d t \leq C_{2} \int_{x_{0}}^{\infty}|h(x)| d x=C
$$

Therefore formula (5) is done.

Corollary 1 Suppose that $h(x)$ is a continuously differentiable function and bounded for all sufficiently large $x \in \mathbb{R}$. If the integrals $\int_{x_{0}}^{\infty}\left|h^{\prime}(x)\right| d x, \int_{x_{0}}^{\infty} p(x) d x$ converge then any solution of the the equation (3) is bounded as $x \rightarrow \infty$.

Proof. From Theorem 1 it follows that the solution $y(x)$ of the equation (3) is bounded and by virtue of the substitution in Lemmal it follows that all solutions of the the equation (2) are also bounded as $x \rightarrow \infty$.

Corollary 2 Suppose that $h(x)$ is a continuously differentiable function and bounded for all sufficiently large $x \in \mathbb{R}$. If the integral $\int_{x_{0}}^{\infty}\left|h^{\prime}(x)\right| d x$ converges and $\int_{x_{0}}^{\infty} p(x) d x=\infty$, then any solution of the equation (2) converges to zero as $x \rightarrow \infty$.

Proof. From Theorem 1 it follows that the solution $y(x)$ of the equation (3) is bounded and by virtue of the substitution in Lemmal it follows that all solutions of the the equation (2) converge to zero as $x \rightarrow \infty$.

Example 3 Consider the equation

$$
z^{\prime \prime}+\frac{2}{x} z^{\prime}+z=\frac{e^{-x} z^{\frac{1}{2}}}{x^{2}}
$$

If we set $z(x)=\frac{y(x)}{x}$ in the equation (6) we obtain

$$
y^{\prime \prime}(x)+y(x)=\frac{e^{-x} y^{\frac{1}{2}}}{x \sqrt{x}}
$$

Here $|h(x)|=\frac{e^{-x}}{x \sqrt{x}} \leq 1$, for all $x \geq 1$

Now we estimate the integral

$$
\begin{aligned}
\int_{1}^{\infty}\left|h^{\prime}(x)\right| d x & =\int_{1}^{\infty}\left|-\frac{3 x^{-5 / 2} e^{-x}}{2}-x^{-3 / 2} e^{-x}\right| d x \leq \\
& \leq \int_{1}^{\infty}\left|\frac{3 x^{-5 / 2}}{2}\right| d x+\int_{1}^{\infty}\left|x^{-3 / 2}\right| d x=3
\end{aligned}
$$

Since the conditions of Theorem 1 holds, the any solution $y(x)$ of the equation (7) is bounded for sufficiently large $\mathrm{x}$. 
Now since the integral $\int_{1}^{\infty} p(x) d x=\infty$, by Lemma 1 it follows that all solutions of the equation (6) converge to zero as $x \rightarrow \infty$.

\section{Unboundedness of Solutions}

Theorem 3 Suppose that $h(x)$ is a continuously differentiable function, bounded for all sufficiently large $x \in \mathbb{R}$, and that the integral $\int_{x_{0}}^{\infty} h^{\prime}(x d x= \pm \infty$ then any solution of equation is (3) unbounded as $x \rightarrow \infty$.

Proof. Since the function $\mathrm{h}(\mathrm{x})$ is bounded then there exists a positive constant $L$ such that $|h(x)| \leq L$ for $\mathrm{x}$ sufficiently large. Assume that the solution $y(x)$ of the equation (3) is bounded for sufficiently large $\mathrm{x}$. It follows that there exists a positive constant $\mathrm{M}$ such that $\max _{x_{0} \leq t \leq x}|y(t)|=M$ for all sufficiently large $\mathrm{x}$.

Multiplying both sides of the equation (3) by $y^{\prime}$ and integrating the result with respect to $\mathrm{x}$ from some positive $x_{0}$ to $x$

$$
0 \leq y^{\prime 2}(x)+y^{2}(x)=y^{\prime 2}\left(x_{0}\right)+y^{2}\left(x_{0}\right)-\frac{2 h\left(x_{0}\right) y^{\alpha+1}\left(x_{0}\right)}{\alpha+1}+\frac{2 h(x) y^{\alpha+1}(x)}{\alpha+1}-\frac{2}{\alpha+1} \int_{x_{0}}^{x} h^{\prime}(t) \cdot y^{\alpha+1}(t) d t
$$

It follows that

$$
y^{\prime 2}\left(x_{0}\right)+y^{2}\left(x_{0}\right)-\frac{2 h\left(x_{0}\right) y^{\alpha+1}\left(x_{0}\right)}{\alpha+1}+\frac{2 h(x) y^{\alpha+1}(x)}{\alpha+1} \geq \frac{2}{\alpha+1} \int_{x_{0}}^{x} h^{\prime}(t) \cdot y^{\alpha+1}(t) d t \geq \int_{x_{0}}^{x} h^{\prime}(t) \cdot y^{\alpha+1}(t) d t
$$

Hence

$$
\left|\frac{2 h\left(x_{0}\right) y^{\alpha+1}\left(x_{0}\right)}{\alpha+1}\right|+\left|y^{\prime 2}\left(x_{0}\right)+y^{2}\left(x_{0}\right)\right|+\left|\frac{2 h(x) y^{\alpha+1}(x)}{\alpha+1}\right| \geq\left|\int_{x_{0}}^{x} h^{\prime}(t) \cdot y^{\alpha+1}(t) d t\right|
$$

Using the Mean Value Theorem for the integral on the right hand side we obtain

$$
A_{x_{0}}+L_{0} M^{\alpha+1} \geq\left|\int_{x_{0}}^{x} h^{\prime}(t) \cdot y^{\alpha+1}(t) d t\right|=\left|y^{\alpha+1}(t *)\right|\left|\int_{x_{0}}^{x} h^{\prime}(t) d t\right|, t * \in\left[x_{0}, x\right] .
$$

Since $\left|\int_{x_{0}}^{\infty} h^{\prime}(t) d t\right|=\infty$, then sending $x \rightarrow \infty$ in the last inequality we get that $M=\infty$. This contradiction proves that the solution $y(x)$ is not bounded as $x \rightarrow \infty$.

Corollary 3 Suppose that $h(x)$ is a continuously differentiable function and bounded for all sufficiently large $x \in \mathbb{R}$. If the integral $\left|\int_{x_{0}}^{\infty} h^{\prime}(t) d t\right|=\infty$ and $\int_{x_{0}}^{\infty} p(x) d x<\infty$, then any solution of the equation (2) is unbounded as $x \rightarrow \infty$.

Proof. From Theorem 3 it follows that the solution $y(x)$ of the equation (3) is unbounded. Since $\int_{x_{0}}^{\infty} p(x) d x<\infty$ then by virtue of the substitution in Lemma1 it follows that all solutions of the equation (2) are unbounded as $x \rightarrow \infty$.

Remark If in the equation (2) the function $P(x)$ is negative then we set $P(x)=-P_{0}(x)$, where $P_{0}(x)>0$, and then all results obtained above will hold.

\section{Special Case of the Equation (2)}

Now consider a special case of nonlinear second-order differential equation

$$
x^{2} z^{\prime \prime}+2 \lambda x z^{\prime}+\left[x^{2}+\lambda(\lambda-1)\right] z=h(x) x^{2+\lambda(\alpha-1)} z^{\alpha}
$$

where $\lambda>0,-1<\alpha<1, \alpha \neq 0$.

Lemma 2 If we let $z(x)=\frac{y(x)}{x^{\lambda}}, \lambda>0$, then the equation (8) is reduced to the equation (3).

Proof. Substituting the expressions $z(x), z^{\prime}(x), z^{\prime \prime}(x)$ in the equation and dividing both sides of equation by $x^{-\lambda}$ we get

$$
x^{2} y^{\prime \prime}+x^{2} y=h(x) x^{2+\lambda \alpha} x^{-\lambda \alpha} y^{\alpha}
$$

Implies that

$$
y^{\prime \prime}+y=h(x) y^{\alpha}
$$

Theorem 4 If the conditions of Theorem 1 hold then any solution of the equation (9) is bounded as $x \rightarrow \infty$. Moreover If the conditions of Theorem 1 hold then any solution of the equation (8) converges to zero as $x \rightarrow \infty$.

Proof. It follows from Lamma 2 and Theorem 1. 
Example 4 Consider the equation

$$
x^{2} z^{\prime \prime}+x z^{\prime}+\left(x^{2}-\frac{1}{4}\right) z=\left(\frac{5 e^{-2 x}+4 e^{-x}+1}{e^{-x}+1}\right) x^{7 l 4} z^{\frac{1}{2}}
$$

Here $h(x)=\frac{5 e^{-2 x}+4 e^{-x}+1}{e^{-x}+1}=5 e^{-x}+\frac{2}{e^{-x}+1}-1, \lambda=\frac{1}{2}, \alpha=\frac{1}{2}$.

Letting $z(x)=x^{-1 / 2} y(x)$, we get

$$
\begin{gathered}
z^{\prime}(x)=-\frac{1}{2} x^{-3 / 2} y(x)+x^{-1 / 2} y^{\prime}(x) \\
z^{\prime \prime}(x)=-\frac{1}{2} x^{-312} y^{\prime}(x)+\frac{3}{4} x^{-5 / 2} y(x)-\frac{1}{2} x^{-3 / 2} y^{\prime}(x)+x^{-1 / 2} y^{\prime \prime}(x) \\
x^{2}\left[\frac{3}{4} x^{-5 / 2} y(x)-x^{-3 / 2} y^{\prime}(x)+x^{-1 / 2} y^{\prime \prime}(x)\right]+x\left[-\frac{1}{2} x^{-3 / 2} y(x)+x^{-1 / 2} y^{\prime}(x)\right]+ \\
+\left[x^{2}-\frac{1}{4}\right] \cdot x^{-1 / 2} y(x)=\left(\frac{5 e^{-2 x}+4 e^{-x}+1}{e^{-x}+1}\right) x^{3 / 2} y^{\frac{1}{2}}
\end{gathered}
$$

We get

$$
y^{\prime \prime}+y=\left(\frac{5 e^{-2 x}+4 e^{-x}+1}{e^{-x}+1}\right) y^{\frac{1}{2}}
$$

Since $h(x)=\left(\frac{5 e^{-2 x}+4 e^{-x}+1}{e^{-x}+1}\right)$ is bounded and the integral

$$
\int_{0}^{\infty}\left|h^{\prime}(x)\right| d x=\int_{0}^{\infty}\left|-5 e^{-x}+\frac{2 e^{-x}}{\left(e^{-x}+1\right)^{2}}\right| d x \leq 7 \int_{0}^{\infty} e^{-x} d x=7
$$

is convergent by comparison test.

So, by Theorem 1 any solution $y(x)$ of the equation (11) is bounded. Hence, by Lemma 2 the solution $z(x)$ of the equation (10) converges to zero. Indeed, the equation has a bounded solution $y(x)=\left(1+e^{-x}\right)^{2}$, as $x \rightarrow \infty$, and the equation has a solution $z(x)=\frac{\left(1+e^{-x}\right)^{2}}{\sqrt{x}}$ which is convergent to zero as $x \rightarrow \infty$.

\section{Acknowledgement}

The author is deeply indebted to the referees for careful and thoughtful reading of the manuscript and useful suggestions which helped to improve the presentation of results.

\section{References}

Ademola, A., \& Arawomo, P. (2011). Stability, boundedness and asymptotic behaviour of solutions of certain nonlinear differential equation. Kragujevac Journal of Mathematics, 35(3), 431-445.

Bucur, A. (2006). About asymptotic behaviour of solutions of differential equations as $x \rightarrow \infty$. General Mathematics, 14(2), 55-58.

Constantin, A. (2000). A note on a second order nonlinear differential system. Glasgow Mathematical Journal Trust, 42, 195-199, UK. http://dx.doi.org/10.1017/S0017089500020048

Hovhannisyan, G. (2004). Asymptotic stability for second order nonlinear differential equations with complex coefficients. EJDE, 85, 1-20.

Kiguradze, I. (1965). The asymptotic behaviour of the solutions of a non linear differential equation of EmdenFowler type. Izv. Akad. Nauk SSSR Ser. Mat, 29(5), 965-986.

Kusano, T., Naito, M., \& Usami, H. (1986). Asymptotic behaviour of solutions of a class of second order nonlinear differential equations. Heroshima mathematical Journal, 16, 149-159.

Lipovan, O. (2003). On the asymptotic behaviour of the solutions to a class of second order nonlinear differential equations. Glasgow Math. J., 45, 179-187. http://dx.doi.org/10.1017/S0017089502001143 
Mingareilli, A., \& Sadarangani, K. (2007). Asymptotic solutions of forced second order differential equations and their extensions. EJDE, 40, 1-40.

Mustafa, O. (2005). On the existence of solutions with prescribed asymptotic behaviour for perturbed nonlinear differential equations of second order. Glasgow Math. J., 47,177-18. http://dx.doi.org/10.1017/S0017089504002228

Saker, S. (2006). Boundedness of solutions second-order forced nonlinear dynamics equation. Rocky Mountain Journal of Mathematics, 36(6), 2027-2039. http://dx.doi.org/10.1216/rmjm/1181069359

Tong, J. (1982). The asymptotic behaviour of a class of nonlinear differential equations of second order. Proceed$\begin{array}{lllll}\text { ings of the } & \text { American } & \text { mathematical } & \text { 84(2), } & \text {. }\end{array}$ http://dx.doi.org/10.1090/S0002-9939-1982-0637175-4

Trench, W. (1976). Asymptotic integration of linear differential equations subject to integral smallness conditions involving ordinary convergence. SIAM J. Math. Anal., 7, 213-221. http://dx.doi.org/10.1137/0507017

Waltman, P. (1964). On the asymptotic behaviour of solutions of a nonlinear equation. Proceedings of the American mathematical society, 15, 918-923. http://dx.doi.org/10.1090/S0002-9939-1964-0176170-8

Wong, J. (1975). Oscillation Theorems for second order nonlinear differential equations. Bull. Inst. Math. Acad. Simca, 3, 283-309. 